\title{
Management of Web Data Models Based on Graph Transformation
}

\author{
Guanglei SONG $^{1}$ Kang ZHANG ${ }^{1}$ \\ ${ }^{1}$ Department of Computer Science \\ University of Texas at Dallas \\ Richardson, Texas 75083-0688 USA \\ \{gxs017800, kzhang, jxk019200\}@utdallas.edu
}

\begin{abstract}
${ }^{*}$
Based on the Reserved Graph Grammar (RGG), this paper presents a unified framework to manage model-based information on the Web in a hierarchical structure. The framework allows models, schemas, and data instances to be represented explicitly and uniformly. The uniform representation of the framework also enables simple user-defined graph transformation rules for different Web data models to translate schemas and data instances between different formats. In addition, the framework implements a set of prototype tools for users to identify meta-primitives at the meta-model level, to define a model or schema by specifying a set of graph grammar rules and to draw the structure of data instances. These features promote a wide scope of Web-related applications, such as information exchange between different organizations, and integration of data coming from heterogeneous information sources.
\end{abstract}

\section{Introduction}

A broad spectrum of data is available on the Web in heterogeneous sources, stored under different formats [Mil98]. Among them, the Extensible Markup Language (XML) has made a rapid advance and great success, meanwhile, many distinct yet highly useful schema languages for XML have been proposed, such as XML Schema [Tho00], RELAX [Mak00], SOX [Dav99] and so on. In spite of the effort of W3C to define a standard for the XML data, a consensus on the most convenient and effective way to represent the structure of XML data is not reached [Tor01]. Web data is not only represented but also defined in different languages. To exchange Web information, two levels of information translation need to be addressed, i.e. data translation for data instances of different formats, and schema translation for schemas among various formats.

A naive way to translate data between different formats is writing a specific translator for each pair of formats. Writing such a program is typically a non-trivial task, and is often complicated by the

\footnotetext{
* The work is partially supported by the National Science

Foundation under grant number IIS-0218738.
}

\author{
Raymond K. WONG ${ }^{2}$ Jun KONG ${ }^{1}$ \\ ${ }^{2}$ School of Computer Science \& Engineering \\ University of New South Wales \\ Sydney, NSW 2052, Australia \\ wong@cse.unsw.edu.au
}

complex manipulation of data sources [Mil98]. A more general framework can be based on a common data model (CDM) [She90] to which the source/target data is mapped, and a common translation language that enables the specification and customization of the translation task. This would facilitate new translations, but still require considerable programming effort whenever a new translation is to be defined [Abi97]. Similarly YAT [Clu98] provides tools for the specification and implementation of data conversions among heterogeneous data sources. It relies on a declarative language, and requires an extra effort for the user to define a translation using the declarative language. In addition to data translation, several techniques have been proposed for schema translation, particularly in the database area [Bat86, Rah01]. These techniques mainly concentrate on restructuring source schemas into other formats rather than data instances.

On the other hand, meta-model based techniques [Atz95, Tor01, Bow02] concentrate on both data and schema translation. A meta-model is a higher-level abstraction of data representation than models. Meta-model based approaches provide a uniform representation for various levels of abstractions and enable data and schema translation declaratively. However, most of the existing approaches are based on textual languages, which are not as intuitive as graph based techniques, such as hyper-graph data model (HDM), and Telos [Myl90]. Graph based techniques construct schema transformation operators, perform inter-model transformations, and define inter-model links in terms of graph. The notations using these techniques are typically defined in a natural language, and thus the ability of automated analysis and transformation of graph-based models is limited. Therefore most of the operations on these data models have to be specified in textual languages. For example, HDM requires considerable effort to specify the transformation rules and links in an appropriative textual language, and Bowers uses RDF [Las99] to represent model-based information translation [Bow02].

This paper presents a unified framework based on graph grammars. In the framework, node-edge diagrams, defined by graph grammars, represent the structure of data models, schemas and data instances. A graph grammar consists of a set of rules (i.e. graph 
transformation rules), which illustrates the way of constructing a complete graph from a variety of nodes. Graph grammars specify all legal inter-connections between individual components, i.e. any link in a valid graph can be eventually derived from a sequence of applications of grammar rules. Graph grammars form a formal basis to represent and transform Web structures in a diagrammatic notation. The framework is based on our recently developed graph grammar formalism, the Reserved Graph Grammar (RGG) [Zha01]. The framework facilitates the following aspects:

- Graphs specify the structure in an intuitive manner. Supported by their theoretical foundation, graph grammars provide a powerful mechanism for automatic syntax checking and verification. Graph transformation rules are also used to specify the transformations of schemas and data instances between different data models. Most current approaches use text or informal graphical notations to specify transformation. Graph transformation rules are, however, intuitive yet formal, and facilitate automation.

- The framework uniformly represents the model-based information on the Web, including models, schemas, and data instances. The uniform representation enables simple user-defined transformation rules for data and the schemas transformations between different models.

The remainder of the paper is organized as follows. It begins with an introduction to the RGG and its capability of representing data models in Section 2, followed by Section 3 that gives an overview of the meta-model framework based on the RGG. Section 4 presents a specification and illustration of the framework by going through an example. Section 5 describes schema and data translation using the meta-model. Finally Section 7 concludes the paper.

\section{The RGG Overview}

The RGG formalism is expressed in terms of diagrams in a node-edge format, which are similar to the "box and line" drawings [All94] but devised to suit automatic analysis based on graph grammars. In a RGG, a node is organized into a two-level hierarchy as illustrated in Figure 1. A large rectangle is the first level called a super-vertex with embedded small rectangles as the second level called vertices. In a node, each vertex is uniquely identified. For convenience and simplicity, the RGG assigns a capital letter to each vertex according to

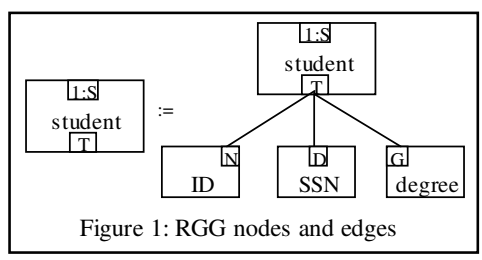

the designer's convention. The name of a super-vertex distinguishes the type of nodes, similar to the type of variables in conventional programming languages. A node can be viewed as a module, a procedure or a variable, etc., depending on the domain requirement and granularity. Edges are used to denote relationships between nodes. Either a vertex or a super-vertex can be the connecting point of an edge. In addition to the structural information, the RGG provides a means of associating data to nodes in terms of attributes. An attribute expresses a piece of data related to the component represented by a node, and can be retrieved and evaluated in the process of parsing.

A RGG consists of a set of graph rewriting rules, also called productions, each having two graphs that are called left graph and right graph as shown in Figure 1. A production can be applied to a given application (called host graph) in the form of an L-application or $R$-application. A sub-graph in the host graph is called a redex if it is isomorphic to the left graph in an $\mathrm{L}$-application or to the right graph in an R-application. An L-application (R-application) to a host graph is to find in the host graph a redex of the left graph (right graph) of the production and replace the redex with the right graph (left graph) of the production. To customize some semantic action, one can write a piece of code (i.e., action) in Java for each graph rewriting rule. An action performs computation over the attributes of a redex when the production is applied. The use of action code in the transformation rules will be discussed in Section 5 .

To allow multiple edge connections to a vertex, we mark the vertex by prefixing its label with a unique integer. The effect of marking a vertex is to preserve the context associated with the vertex during transformation.

\section{The Framework Overview}

Information of interest in this paper consists of three levels: models, schemas, and instances [Bow02] as shown in Figure 2. At the bottom level instances contain concrete Web data, such as XML, HTML documents. At the second level, a schema defines the structure of instances. Different schemas are defined by different formalisms, called models at the third level. Each level of the architecture can be viewed as an instantiation of the level above. More specifically, models are particular instantiations of the abstractions defined by a higher-level model, schemas are particular instantiations of a model, and instance-level data are instantiations of a schema. Apart from the three levels, to define multiple data models, we provide a level of abstraction above the model, called meta-model, which defines the basic 
abstractions used to define models. In the following, the term primitive refers to a component of a model, and the term meta-primitive refers to a component of a meta-model.

To describe complex structures intuitively and perform transformation rules effectively, the RGG is adapted with the following extensions:

1. The algorithm to search for an occurrence (i.e. a redex) of a right graph (in R-application) or a left graph (in L-application) in the host graph is adjusted to search for a newly defined redex. The redex is redefined as follows: a redex is a sub-graph of the host graph, which is an instance the right graph (in R-application). A right graph could match multiple types of redexes instead of only one in the previous version of the RGG. For the example rule in Figure 1, if the node degree connects to the node student with a cardinality of 1 to 5 , then the right graph could have five possible redexes, which have different numbers of degree nodes.

2. Different from the process of validation, in which the parser finally presents a parsing result, i.e. valid or not, the transformation process produces a new graph from the input host graph according to the transformation rules. In order to perform the transformation rules, we add another parser to the framework, called transformer. Slightly different from the original parser, the transformer performs the embedding process in a copy of host graph and adjusts the copy to a new graph as the result.

3. Like the output of a validation process, "valid" or "invalid", different from that of a transformation, the termination condition of graph transformation is different from that of validation. The transformation process terminates when every node is parsed or no more redex, while in the validation process the parser terminates only when the host graph is reduced to a null graph (usually denoted as $\lambda$ ) or no more redex in the host graph.

Using the RGG as a meta-model, this paper proposes unified framework for data representation and transformation.

Nodes and edges in the RGG represent meta-primitives and relationships respectively. A node denotes a component of a model, a schema or an instance. Labeled with different names, nodes have different semantics in different contexts, e.g. an element of Schema, or a PCDATA of DTD. An edge defines a relationship between two nodes, such as element-element relationship and element-attribute relationship. Each RGG rule denotes relationships among components. A whole set of rules consisting of meta-primitives defines a model, that consisting of primitives defines a schema, and that consisting of instance constructs defines an instance. Figure 3 shows the hierarchical abstraction levels of meta-model, model,

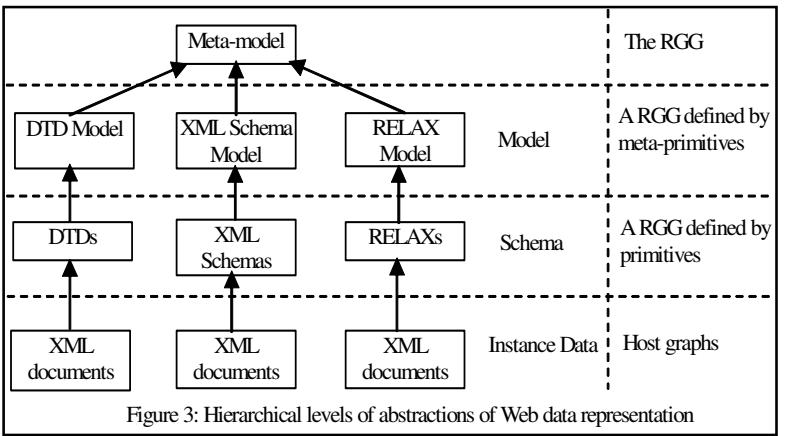

schema, and instance data on Web, and their correspondences with the formalisms in the RGG. As a meta-model, the RGG enables users to define a model as a RGG, i.e. a set of graph transformation rules. A schema is defined by a RGG, which is a host graph of the RGG at the model level in terms of primitives. A data instance is denoted by a host graph of the RGG at the schema level.

Figure 4 shows the architecture of the framework, which consists of three major modules, the visual object generator (VOG), the rule generator, and the visual modeling environment (VME), which is automatically generated.

VOG is used to define meta-primitives. The module provides a generic approach for users to introduce a new meta-primitive suitable for the meta-model when a new model needs a special construct that has no counterpart in the meta-model. Atzeni et al. considered this kind of approach being "asymptotically" complete [Atz95] in the sense of generality and variety of meta-models. Whereas they did not provide facility for users to conduct the extension, we present the visual editor, i.e. VOG, to facilitate the process.

The rule generator is a visual environment for users

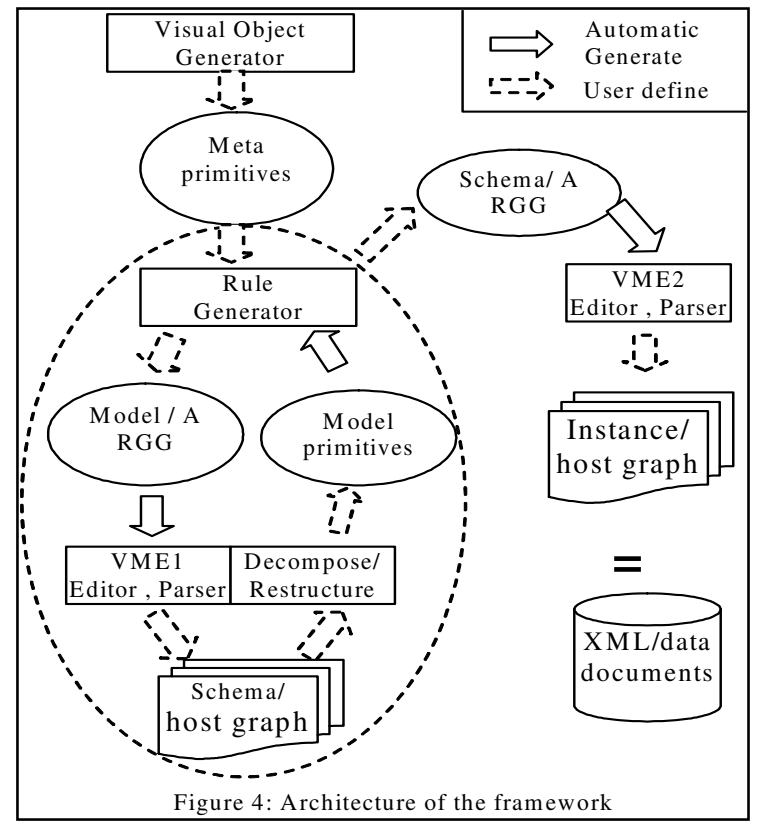


to define graph transformation rules. With user-defined meta-primitives or primitives, a user may construct a graph grammar to define a model or a schema according to different types of components. Supplied with user-defined rules, the rule generator compiles and automatically generates a visual modeling environment (VME).

Each VME consists of a graph editor and a parser for the pre-defined graph grammar, based on which the VME is generated. The graph editor provides users with guidance on how to draw a host graph to represent a schema (VME1 in Figure 4) or a data instance (VME2), and prompts errors whenever the syntax is violated. It is also able to perform syntax-directed computations, specified with individual productions.

To use the framework to specify and transform data or schema, users perform the following steps as illustrated in Figure 4:

1. define meta-primitives that are able to capture the main primitives adopted by different schema languages for structured data (XML documents in particular) over the Web;

2. define a RGG of interest by using the meta-primitives, i.e. a model, based on which users further define schemas and transformation rules; and

3. define source data instances and automatically apply the rules.

As shown in Figure 4, the second step could become an infinity loop, which simulates the recursive definition in the real world. For example, the XML Schema is used to define XML documents, while it is an XML document itself, i.e. schema is an instance of its own.

\section{Model-Schema-Data Specification}

This section presents the model-schema-data specification and shows the basic process of data model management by identifying meta-primitives of a meta-model, defining a model, constructing a schema, and drawing an instance.

\subsection{Identifying Meta Primitives}

All the constructs used in most known models and formalisms for expressing Web data fall into a rather limited set of categories [Zha01]. Therefore a meta-model can be defined by means of a basic set of meta-primitives, corresponding to these categories.

In considering the above fact, our example meta-model includes the meta-primitives limited to the following: object type, ordered sequence, unordered sequence, choice, cardinality, key, and foreign key. Obviously the meta-primitives are not complete for defining all the Web data models, so the names and number of nodes may change when the meta-model needs to be extended to subsume newly introduced models. To ease the process of extending the meta-model, our framework provides the VOG for users to specify new meta-primitives. A relationship between meta-primitives is defined by an edge, whose semantics is determined by the nodes it

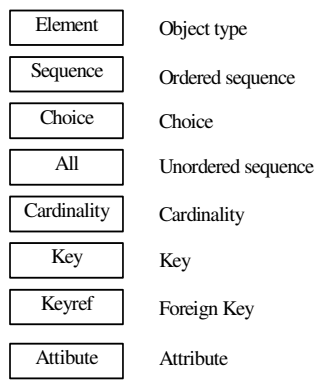

Figure 5: Meta primitives of the metamodel connects. The set of example meta-primitives includes eight types of nodes (meta-primitives) as shown in Figure 5.

\subsection{Defining a Model}

This section uses the meta-primitives in Figure 5 to define an example model for a subset of the XML schemas, which includes most of XML Schemas and DTDs. In addition, our framework provides the rule generator as a convenient way for users to define and add more rules to specify a complete set of schemas for the structure of XML documents. Figure 6 shows the model defined by a graph grammar, which consists of 14 rules, and the $\mathrm{i}$ th production is marked with $<i>$.

The model defines relationships among Elements and Attributes etc. Rules $<1>$ and $<5>$ declare that an element can enclose arbitrary number of other Elements and Attributes respectively. Rule <2> defines that an Element may have a Cardinality constraint. Rules $<4>$ and $<8>$ define that an Element may have an arbitrary number of Keys or Foreign Keys. Rules $<7>,<11>$, and $<14>$ define that an Element may have a Sequence, a Sequence may include any number of Elements, and a Sequence may have a Cardinality constraint, respectively. Rules $<10\rangle,\langle 12>$, and $<13>$ define the same relationships

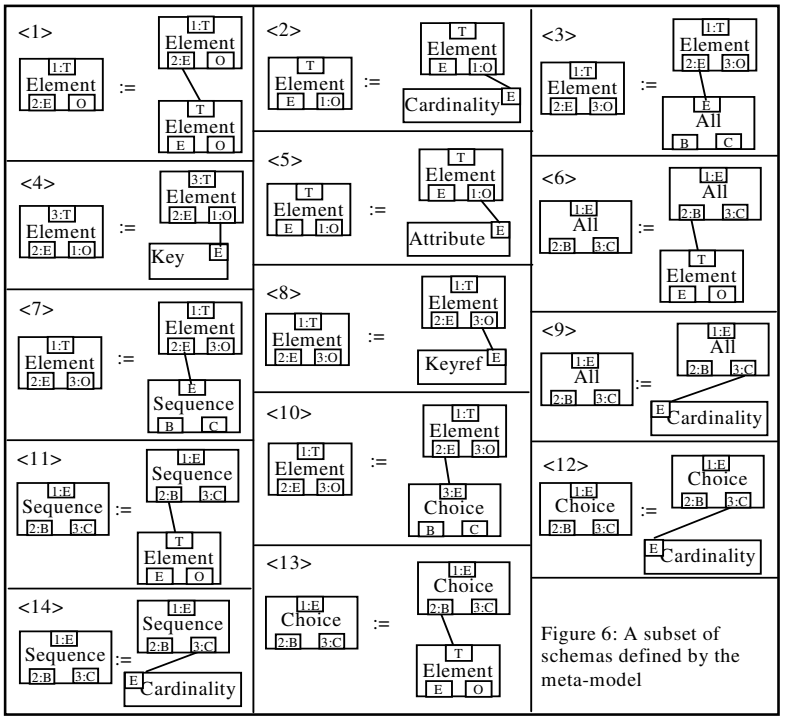


among Elements, Cardinalities, and Choices. Rules $<3>,<6>$, and $<9>$ define the relationships among Elements, Cardinalities, and All.

\subsection{Constructing a Schema}

After the rule generator parses the rules, i.e. the model, a VME is automatically generated. Using the VME, the user draws and customizes a host graph, i.e. an instance of the model, which defines a schema. Then with user intervention, the VME transforms the host graph into a set of rules suitable for the system to define the structure of data instances. During the procedure users should interact with the system to adjust the schema, i.e. the procedure is semi-automatic. To construct a schema using VME, users should follow the three steps described in the following three subsections.

\subsubsection{Drawing a Host Graph.}

The user draws a host graph in the generated VME to represent the structure of the expected schema. Figure 7 shows an example of the host graph conforming to the rules described in Figure 6. The host graph defines the structure of a schema, in which the name of each node denotes the type of the node, such as Element and Sequence. The host graph does not however define the instance of the type, such as the name of an element or range of the cardinality, which is domain specific. The framework cannot determine the value of each node automatically. Therefore the host graph does not completely define the structure of documents, and rest of the job has to be tackled by the user, i.e. to customize the host graph in the VME.

\subsubsection{Customizing the Host Graph.}

To customize the host graph means to specify the parameters of each node according to the domain requirement, such as the name of an element, the range of a cardinality, and so on. This could be done very easily with the generated VME by editing each node. Figure 8 shows the user-defined domain specific

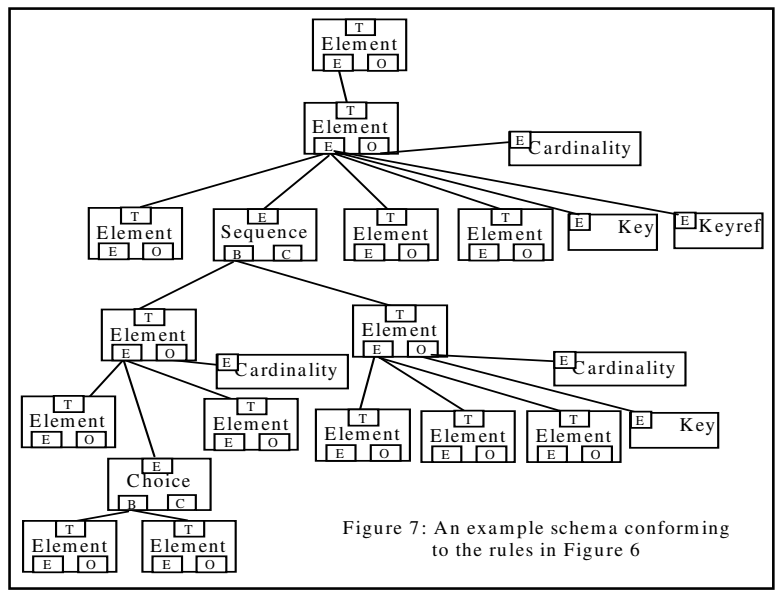

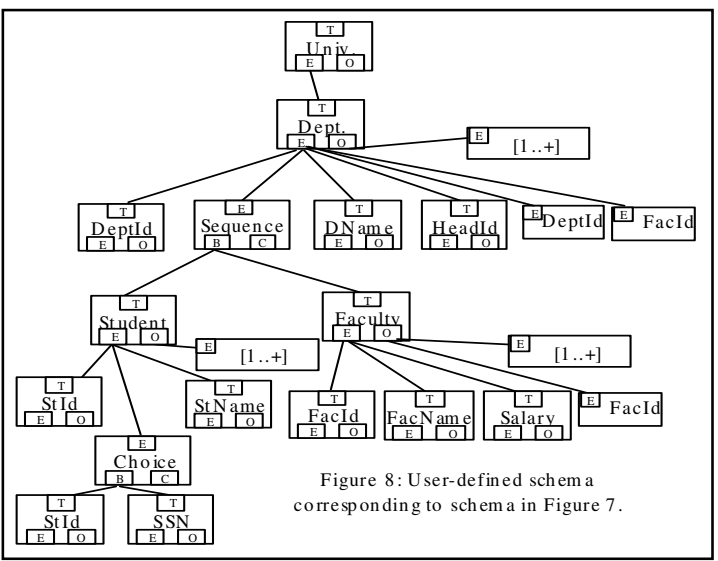

schema based on the example in Figure 7. The name of the node Sequence is the same as in Figure 7, but the meaning is different, the one in Figure 7 defines the type of the node, while the Sequence in Figure 8 represents the instance itself. The DTD defines a Sequence using a comma "," between every two elements.

\subsubsection{Adapting the Rules.}

After being customized in the domain context, the host graph represents the structure of a schema. From now on we refer to the host graph as schema graph. Before the rule generator processes a schema graph, it needs to be adapted. 1: Although we can simply make the schema graph a right graph of a rule, we still need a left graph, because each rule of a graph grammar consists of a right graph and a left graph. 2: As some nodes in the host graph carry structural semantics, they should be converted to the corresponding notations in the RGG for the parser to recognize. 3: The number of nodes in the right graph influences the performance of the parsing process. The larger the right graph is, the more costly the parsing process is. So a large schema graph should be broken into smaller ones to improve the parsing performance. 4: In order to further construct rules for data translation based on the schema, the schema graph needs to be decomposed. The rules for translation will be addressed in more details in Section 5.

The VME automatically adapts the schema according to the following principles.

1. Remove those nodes that carry structural semantics, such as cardinality and attribute, and add appropriate attributes to the nodes associated with the removed nodes.

2. Trim vertices of each super-vertex, i.e. remove those vertices not used in the schema, such as the vertex links to the cardinality node.

3. Break any schema trees of more than three levels to smaller trees to improve the parsing performance.

4. For each sub-graph of the schema graph, add the left graph to construct a rule. 


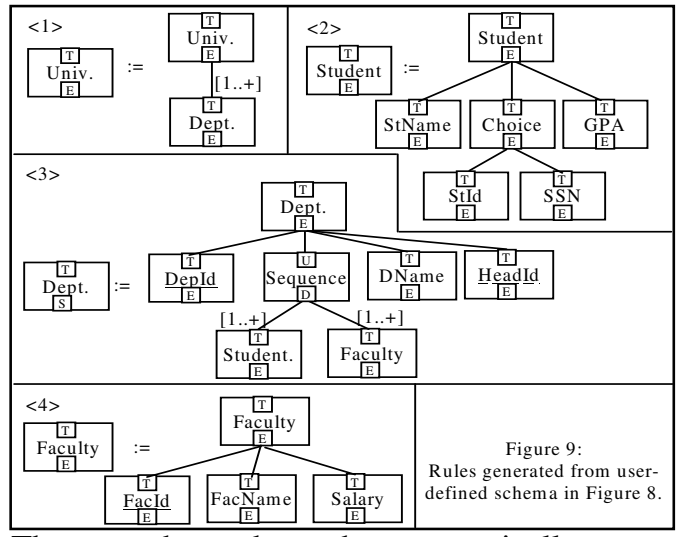

The user then adapts the automatically generated rules in the rule generator. The rule generator could in turn parse the rules and generate a new VME for the user to draw instance documents. Figure 9 shows the resulting rules generated from the user-defined schema in Figure 8. In the generated VME, the user could draw and parse host graphs, which are instances of the schema.

\subsection{Drawing an Instance}

The VME generated by the rule generator from a schema graph enables users to visually define any instance documents conforming to the schema.

Figure 10 illustrates an example document conforming to the schema rules defined in Figure 9. In the VME, a user can draw any host graph, which defines the structure of an instance document and conforms to the rules used to generate the VME, i.e. the schema. So far, the host graph does not have concrete data except the structure. The framework cannot determine what the user wants data to represent; therefore the user has to customize the data instance in the structure.

In the host graph, the name of each node is a markup in a markup language, which denotes the data type of the node and could enclose an instance value. For example, StName in Figure 10 is of data type "string", and could have any string as a value, such as "Lawrence". For simplicity, Figure 10 does not show the detailed value of each node. After the user instantiates the structure, the document is completed.

Recall the process of constructing a schema, we find that the process of drawing an instance has a uniform procedure except that the host graph of an instance needs not to be adapted for further definition.

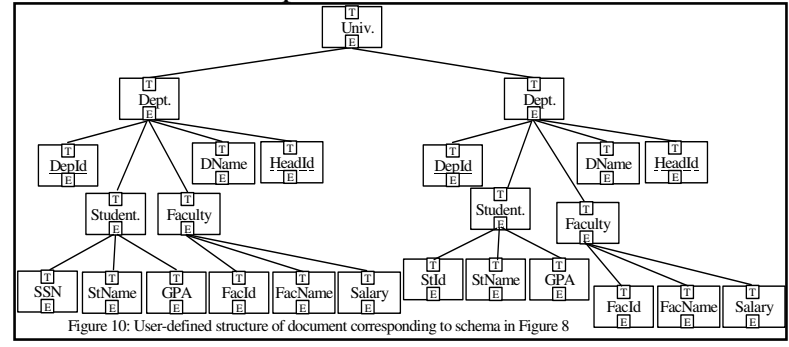

\section{Inter-Model Transformation}

By going through an instance transformation example in the unified data representation framework, this section illustrates a uniform specification for defining inter-model transformation. Due to space limitation, only the data translation is presented. It is, however, easy to understand that similar transformation rules can be defined for schema translation (using the constructs presented in the previous sections).

\subsection{Inter-Model Transformation Specification Overview}

A transformation specification consists of a set of transformation rules, each consisting of a left graph and a right graph. The left graph of a transformation rule defines the structure of the resulting graph. In the framework each model or schema is defined by a set of RGG rules, from which graph transformation rules are extended by adapting the left graph of a rule for the goal of transformation on the right graph.

The graph transformation rule shown in Figure 11 explicitly and precisely define the syntax of instance graphs when the sub-graph with dotted lines are excluded; and the mechanism for transforming the email syntax in the right graph to the email syntax in the left graph when the sub-graph with the dotted lines are included. We refer to the former rule as grammar (email with subject and message), and the latter, extended from the grammar, as translator (from the structure of email with subject and message to that with head and body). Figure 11 shows both the grammar and the translator together (differentiated by dotted lines).

Except defining a different set of rules, the steps of transforming a data instance or a schema are similar to that of defining a schema. The following two sections briefly go over two examples to illustrate the transformation process.

\subsection{An Instance Transformation Example}

Figure 12(b) shows the structure of a source XML document, which defines a page with two sections. To transform it to a WML document, one defines the transformation rules inside the translation rule generator shown in Figure 12(a). The translator consists of 7 translation rules, and each having a left graph and a right graph. In the VME generated according to the translator, the host graph in Figure 12(b) is automatically transformed to a WML document as shown in Figure 12(c).

We can define transformation

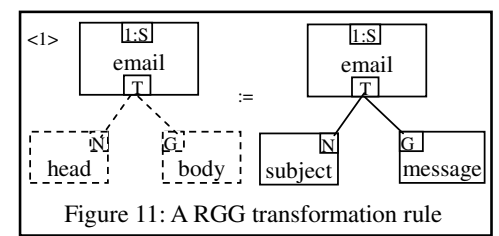




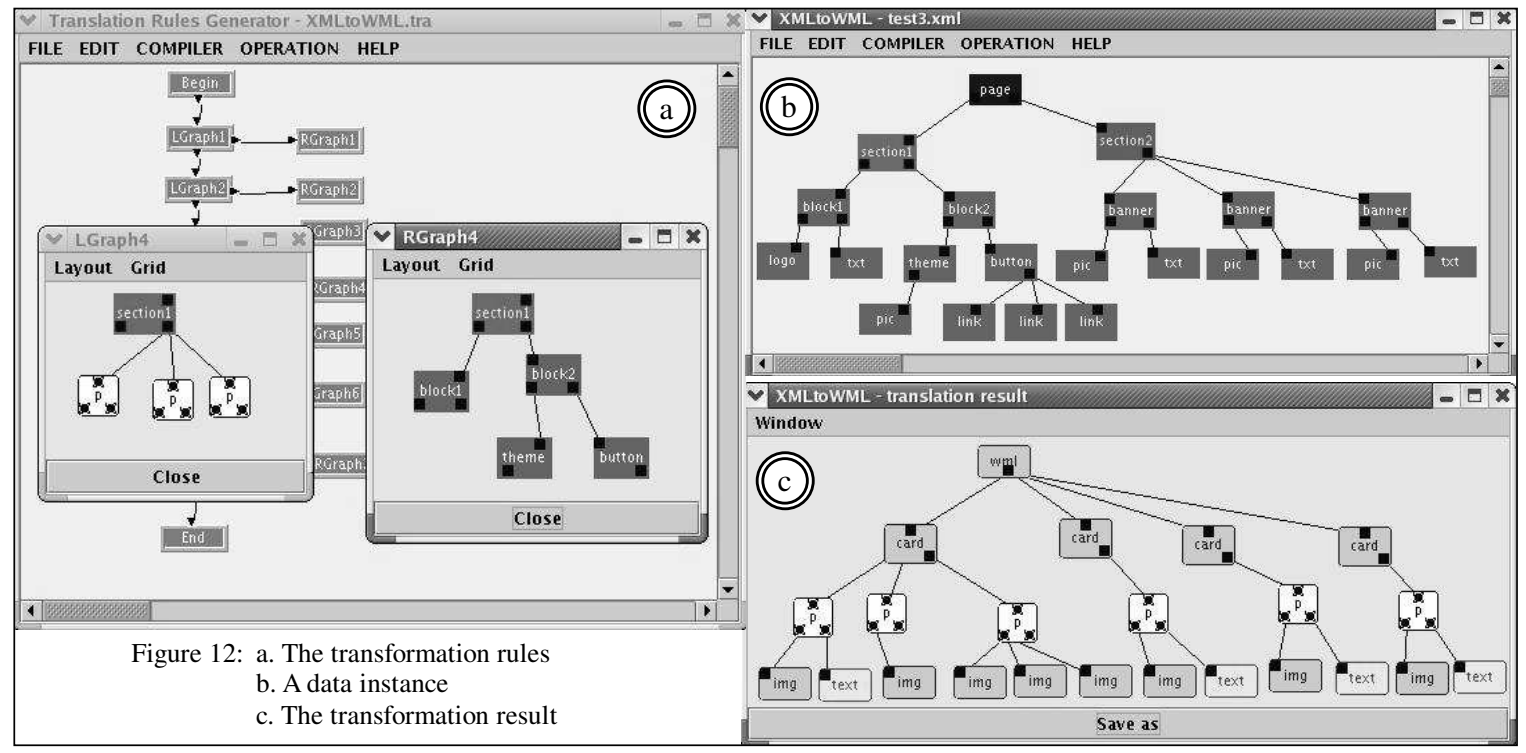

rules to translate between any two schemas using the rule generator in a similar fashion. Under the uniform framework and meta-model, it is intuitive and straightforward to define different transformation rules.

\section{Conclusion}

Based on the adapted RGG, this paper presented a unified framework for management of Web data models. We have already implemented a set of prototype tools based on the adapted RGG to facilitate the management of Web data except that the adjusted algorithm is still under development. With the tools, we specified an example of data translation and verified that the framework is practically feasible.

We believe that the research presented contributes not only to model development for Web data, but also to any data integration and information exchange over the Internet. We are currently investigating the applicability of graph transformation to generic model management through model operators and making encouraging progress [Son04]. As for the future work, we will extend the meta-model to include various database models and semi-structured Web data models and to provide a solid prototype system to boost the productivity of generic model management.

\section{References}

[Abi97] S. Abiteboul, S.Cluet, and T. Milo. Correspondence and Translation for Heterogeneous Data. Proceedings of International Conference on Data Theory (ICDT), 1997.

[All94] R. Allen and D. Garlan. Formalizing Architectural Connection, Proceedings of 16th International Conference on Software Engineering, pp.71-80, 1994.

[Atz95] P. Atzeni and R. Torlone. Schema Translation Between Heterogeneous Data Models in a Lattice Framework. Proceedings of Sixth IFIP TC-2 Working Conference on Data Semantics (DS-6), Atlanta, Georgia, 1995.

[Bat86] C. Batini, M. Lenzerini, and S. B. Navathe. A Comparative Analysis of Methodologies for Database Schema Integration. ACM Computing Surveys, 18(4): 323-364, December 1986.

[Bow02] S. Bowers and L. Delcambre. A Generic Representation for Exploiting Model-Based Information. ETAI Journal, Linköping Electronic Articles in Computer and Information Science, Vol. 6(2001), March, 2002.

[Clu98] S. Cluet, C. Delobel, J. Simeon, and K. Smaga. Your Mediators Need Data Conversion! ACM SIGMOD, pp.177-188, 1998.

[Dav99] A. Davidson, M. Fuchs, M. Hedin, M. Jain, J. Koistinen, C. Lloyd, M. Maloney, K. Schwarzhof. Schema for Object-Oriented XML 2.0. W3C Document, July 1999.

[Las99] O. Lassila and R. R. Swick. Resource Description Framework (RDF) Model and Syntax Specification. W3C Recommendation, February 1999.

[Mak00] M. Makoto. RELAX (REgular LAnguage Description for XML). Internet Document, April 2000. (http://www.xml.gr.jp/relax/).

[Mi198] T. Milo and S. Zohar. Using Schema Matching to Simplify Heterogeneous Data Translation. Proceedings of Very Large Databases (VLDB), New York, August 1998.

[My190] J. Mylopoulos, A. Borgida, M. Jarke, and M. Koubarakis. Telos: Representing Knowledge About Information Systems. ACM Transactions on Information Systems, 8(4):325-362, October 1990.

[Rah01] E. Rahm and P. A. Bernstein. A Survey of Approaches to Automatic Schema Matching. The VLDB Journal 10: 334-350, 2001.

[She90] A. Sheth and J. Larson. Federated Database Systems. ACM Computing Surveys, 22 (3): 183 - 236, 1990.

[Son04] G.L. Song, K. Zhang, and J. Kong. Model Management Through Graph Transformation. Proceedings of 2004 IEEE Symposium on Visual Languages and Human-Centric Computing (VL-HCC'04), Rome, Italy, 26-29 September 2004.

[Tho00] H. S. Thompson, D. Beech, M. Maloney, and N. Mendel-Sohn (Eds.). XML Schema Part 1: Structures. W3C Document, April 2000.

[Tor01] R. Torlone and P. Atzeni. A Unified Framework for Data Translation over the Web. Second International Conference on Web Information System Engineering (WISE'2001), Kyoto, Japan, IEEE Computer Society Press, 2001.

[Zha01] D. Q. Zhang, K. Zhang, and J. Cao. A Context-Sensitive Graph Grammar Formalism for the Specification of Visual Languages. The Computer Journal, 44(3), pp.187-200, 2001. 